\title{
Deformation Behavior of Press Formed Shell by Indentation and Its Numerical Simulation
}

\author{
Minoru Yamashita and Zubair Bin Khalil \\ Department of Mechanical Engineering, Gifu University, 1-1 Yanagido, Gifu 501-1193, Japan \\ Correspondence should be addressed to Minoru Yamashita; minoruy@gifu-u.ac.jp
}

Received 16 June 2015; Accepted 21 September 2015

Academic Editor: Yuanxin Zhou

Copyright (C) 2015 M. Yamashita and Z. Bin Khalil. This is an open access article distributed under the Creative Commons Attribution License, which permits unrestricted use, distribution, and reproduction in any medium, provided the original work is properly cited.

\begin{abstract}
Deformation behavior and energy absorbing performance of the press formed aluminum alloy A5052 shells were investigated to obtain the basic information regarding the mutual effect of the shell shape and the indentor. Flat top and hemispherical shells were indented by the flat- or hemispherical-headed indentor. Indentation force in the rising stage was sharper for both shell shapes when the flat indentor was used. Remarkable force increase due to high in-plane compressive stress arisen by the appropriate tool constraint was observed in the early indentation stage, where the hemispherical shell was deformed with the flat-headed indentor. This aspect is preferable for energy absorption performance per unit mass. Less fluctuation in indentation force was achieved in the combination of the hemispherical shell and similar shaped indentor. The consumed energy in the travel length of the indentor equal to the shell height was evaluated. The increase ratio of the energy is prominent when the hemispherical indentor is replaced by a flat-headed one in both shell shapes. Finite element simulation was also conducted. Deformation behaviors were successfully predicted when the kinematic hardening plasticity was introduced in the material model.
\end{abstract}

\section{Introduction}

Automobile body usually consists of many sheet metal components. Most of them are usually fabricated by various press forming operations from sheet metal. They are often joined by resistance spot welding or other welding processes, bolt and nut, adhesives, and so forth. Automobile body designers consider not only the strength for durability but also structural ability to protect the occupants during impact by collision event. The acceptable limit in acceleration acting on the human body has to be satisfied in the passive safety concept. Further, the cabin deformation by crash should be kept under a certain level. Sheet metal members are plastically collapsible to play the important role as shock absorber, which dissipates the kinetic energy.

The body deformation by collision is crumple, whose pattern is complicated, because the plastic deformation in many components occurs at same time and then their mutual interaction is inevitable. Also for the aircraft fuselage, helicopter body, and train body, the energy absorbing structural design is adopted for protection of occupants.

For several decades, increasing concerns have been paid with respect to the shock absorbing deformation of thinwalled metal structures. Review papers discussing the collapse deformation were published, which covers a wide range of thin-walled structures, for example, circular or square tubes, frusta, struts, and honeycombs [1,2]. As a practical example, crush deformation of the helicopter subfloor structure assembled with sheet metal parts by riveting was studied for improvement of the energy absorption capability [3]. Further, constitutive modeling for prediction of material failure was described for crashworthiness, where the shell element splitting techniques were demonstrated for crack propagation [4].

Dome shaped or hemispherical shells were also the energy absorbers. Plastic collapse of aluminum egg-box panels subjected to out-of-plane compression was investigated. Collapse strength and energy absorption are sensitive to the 
level of in-plane constraint [5]. Aluminum shells with various radius/thickness ratios were compressed by two rigid plates under low speed condition. An analytical model was also proposed to predict the compressive load curve [6]. Spherical shells were crushed by a drop-hammer to investigate the energy absorption capacity. The numerical simulation was also performed. Their dynamic behavior was compared with quasistatic one $[7,8]$.

Hemispherical metal domes were deformed by indenting several kinds of solid bars or tubes whose tip shape was flat, round-headed or notched, and so forth. The effect of the indentation manner on the collapse load curve was examined in both experiment and finite element method $[9,10]$. For collapsible energy absorbers, inversion and reinversion deformations of frusta were found very appropriate, where the accumulated absorbed energy per unit mass and the usability of the absorber several times were considered [11]. Honeycombs are also widely used as the shock absorbers. Impact experiment for various cell shapes and the corresponding numerical simulation were carried out. The numerical model considering the cyclic symmetry was constructed [12].

For the frontal crash safety of automobile, many investigations were performed for the collapse deformation of tubular structures. The deformation of tubular structures is sensitive for the geometries, dimensions, supporting condition, and so forth. Axial compressive deformation of circular and square steel tubes was theoretically well predicted [13]. Collapse of tubular structures with hat cross-section assembled with rivets, screws, or/and adhesive was experimentally investigated [14]. The numerical model of hat tube was developed for the adhesive bonded one. The effect of the strength of the adhesive was demonstrated showing good agreement with the corresponding experiment [15].

The present study is focused on the energy absorbing performance of the press formed shell. The objective is to obtain the basic information in energy absorbing deformation behavior for optimizing the design of the collapsible components, for example, in automobile and train bodies, building, and structures. The shapes of the press formed shell are flat top and hemispherical. The material was aluminum alloy A5052-H34 sheet. Indentation was conducted using flator hemispherical-headed indentor. Numerical simulation was also carried out by the dynamic explicit finite element method in order to present appropriate numerical model to achieve accurate prediction.

\section{Test Material and Experimental Conditions}

The test material is a commercially available aluminum alloy A5052-H34 sheet with $1 \mathrm{~mm}$ thickness. The mechanical properties are listed in Table 1. Specimens are the flat top and the hemispherical shells. They are formed from a circular blank with $80 \mathrm{~mm}$ diameter using a hydraulic deep-drawing testing apparatus illustrated in Figure 1. The formed height was set to $10 \mathrm{~mm}$. Forming operation was carried out under quasistatic condition, where the punch speed was about $0.1 \mathrm{~mm} / \mathrm{s}$. A commercially available lubricant (CD400, Daido chemical industry) for press forming was applied to all tool-material interfaces. The blank holding force was $3.5 \mathrm{kN}$ which was
TABLE 1: Mechanical properties of test material.

\begin{tabular}{lcccc}
\hline Material & $C[\mathrm{MPa}]$ & $n$ & U.T.S. [MPa] & $E[\%]$ \\
\hline A5052-H34 & 383 & 0.15 & 248 & 8.3 \\
\hline
\end{tabular}

Plastic property: $\sigma=C \varepsilon^{n}$, U.T.S.: ultimate tensile strength, and $E$ : total elongation.

twice of the value calculated by Siebel's formula [16]. It gives the minimum blank holding force without flange wrinkling.

The tip shapes of the indentor are flat-headed and hemispherical. The combinations of the shell and the indentor are shown in Figure 2. The abbreviated notations FS, FF, SS, and SF are used for the combinations. The first letter represents the shell shape, F: "F"lat top or S: hemi “S"pherical shell. The latter represents the indentor, F: "F"lat-headed or S: hemi "S"pherical-headed indentor. The indenting operation was also performed using the deep-drawing testing apparatus by substituting the indentor for the forming punch. The indentation speed was about $0.1 \mathrm{~mm} / \mathrm{s}$.

\section{Experimental Results and Discussions}

Relationship between the collapse stroke and the force is the most important characteristic in designing the press formed product as effective energy absorber. Figure 3 demonstrates the progressive deformation patterns for 4 kinds of experimental conditions. The corresponding indentation force curves are shown in Figure 4.

Deformation behavior of the flat top shell is first discussed. The hemispherical indentor gradually contacts the flat top part of the shell; thus the concavity is formed on its top in FS condition. The indentation force gradually increases in the early deformation stage. The portion of the shell that was in contact with the die profile during press forming is unbent or flattened. On the other hand, the hat top corner portion contacted with the punch profile during forming decreases the radius. The indentation force once drops and takes minimum at around $22 \mathrm{~mm}$ indentation stroke, because the part outside the indentor becomes flat. Then the geometrical rigidity becomes low. The indentation force shows a rapid increase, when the shell wall deformation is extensional. Since the deformation behavior depending on the shell shape and the indentation condition is the exclusive concern in the study, the deformation in the extensional stage is excluded, though the energy is consumed in the deformation.

As for the FF condition, the force exhibits a steeper slope than that of FS in the early stage of indentation. The main deforming zone is the vicinity of the round corner of the indentor in the early stage, where the deformation is more extensive in comparison with that in FS. The maximum indentation force is almost comparable to that in FS. The indentation stroke at force drop is shorter in FF than that in FS. Finally, drop and steep increase of the force also appear by the same reason mentioned above.

Next, deformation of hemispherical shell specimen is discussed. In the case of SS condition, contact area increases with the increase of indentation force. The plastic hinge moves outward according to the contact position to the 


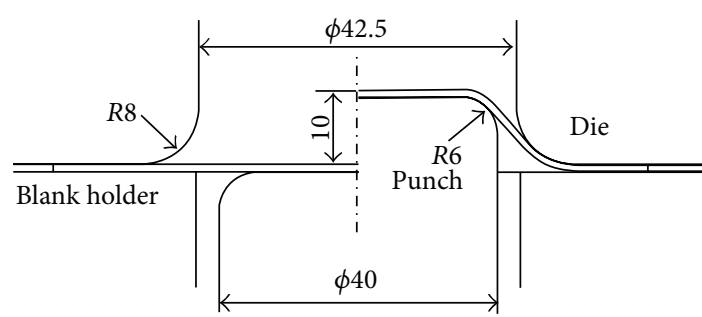

(a) Flat top shell specimen

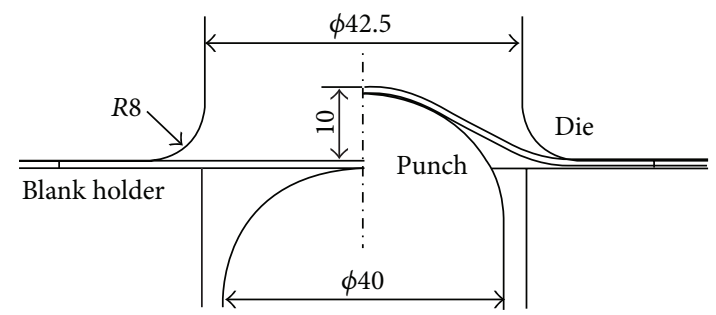

(b) Hemispherical shell specimen

FIGURE 1: Sheet drawing process.

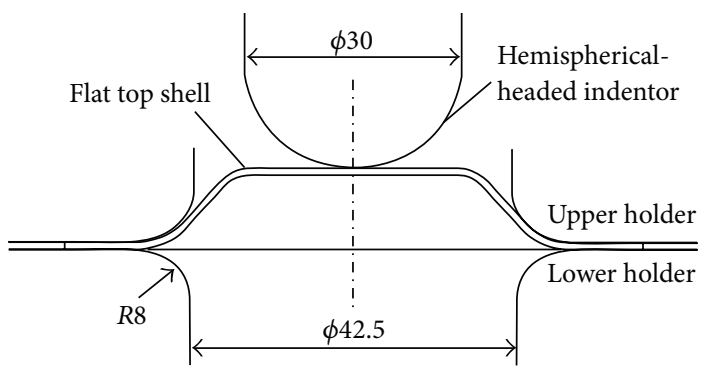

(a) FS: flat top shell and hemispherical-headed indentor

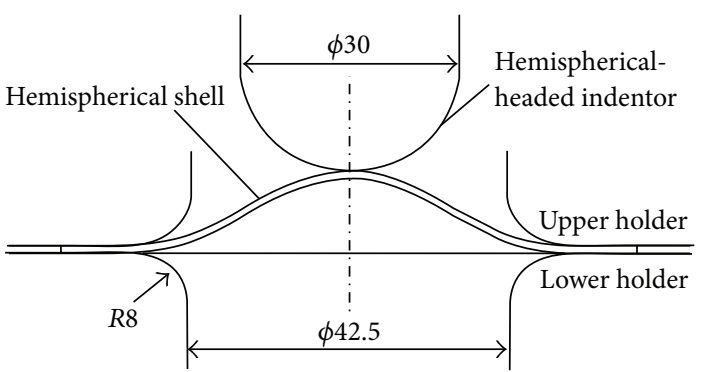

(c) SS: hemispherical shell and hemispherical-headed indentor

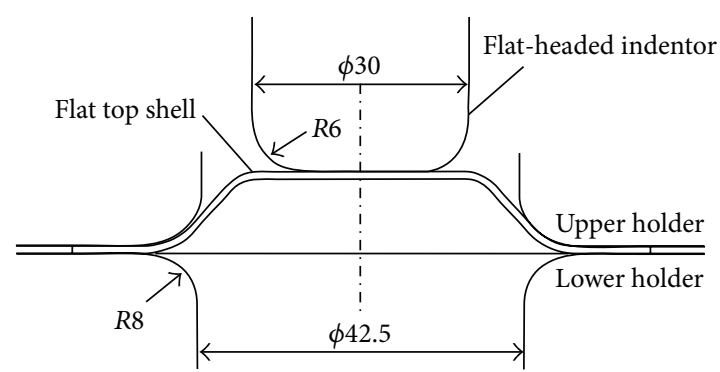

(b) FF: flat top shell and flat-headed indentor

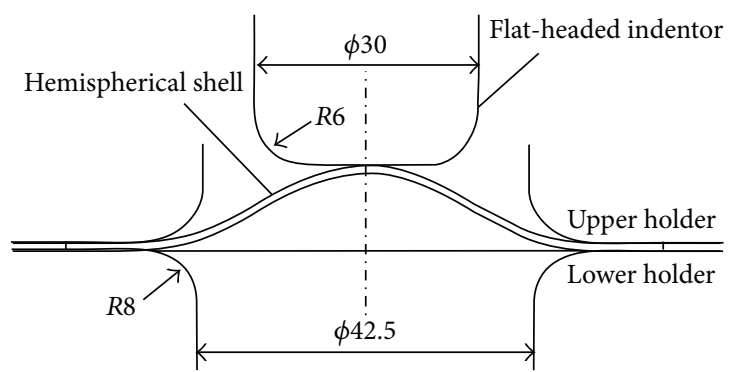

(d) SF: hemispherical shell and flat-headed indentor

FIGURE 2: Variation in combinations of formed shells and indentors.

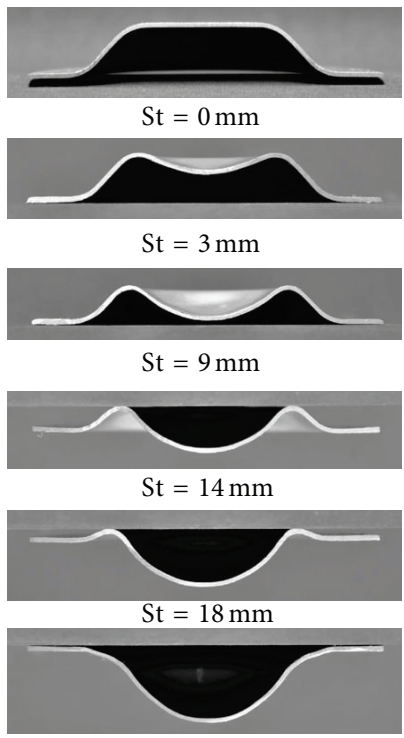

$\mathrm{St}=23 \mathrm{~mm}$

(a) FS
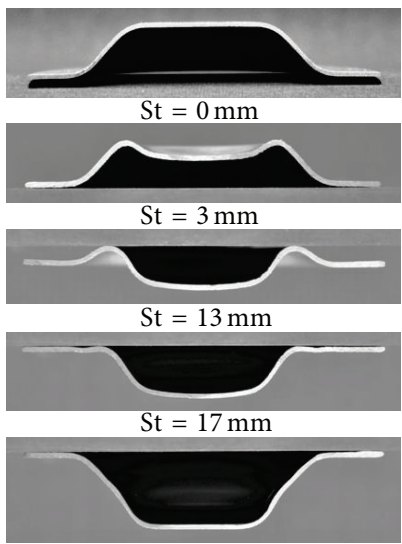

St $=21 \mathrm{~mm}$

(b) FF

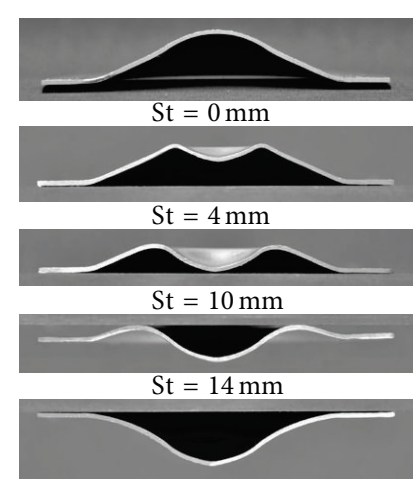

St $=19 \mathrm{~mm}$

(c) SS

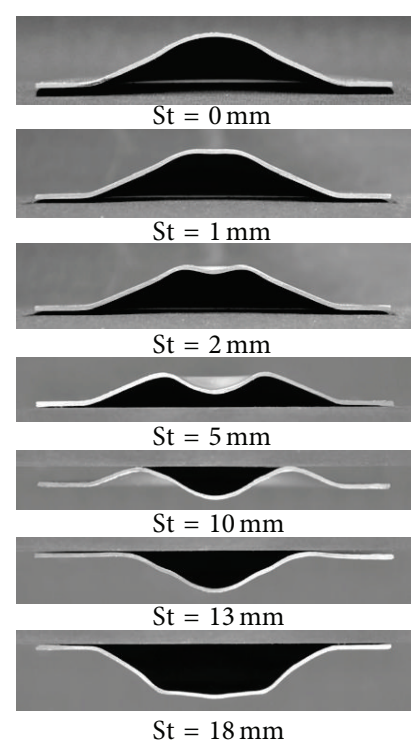

(d) $\mathrm{SF}$

FIGURE 3: Progressive deformation patterns under various indentation conditions (St: indentation stroke). 


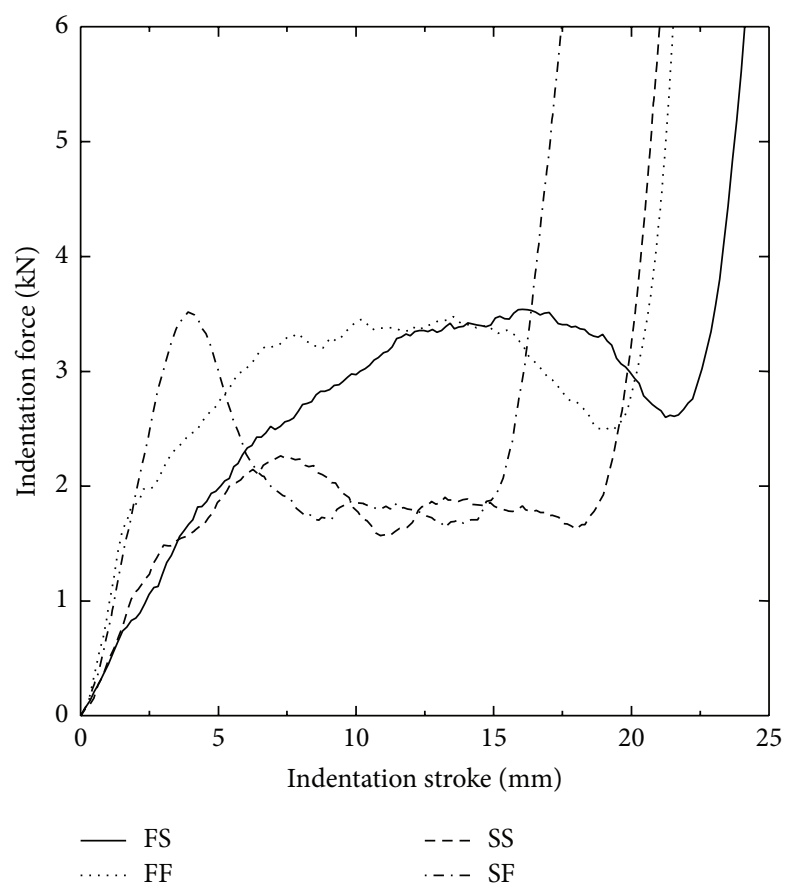

FIGURE 4: Indentation force curves under various indentation conditions.

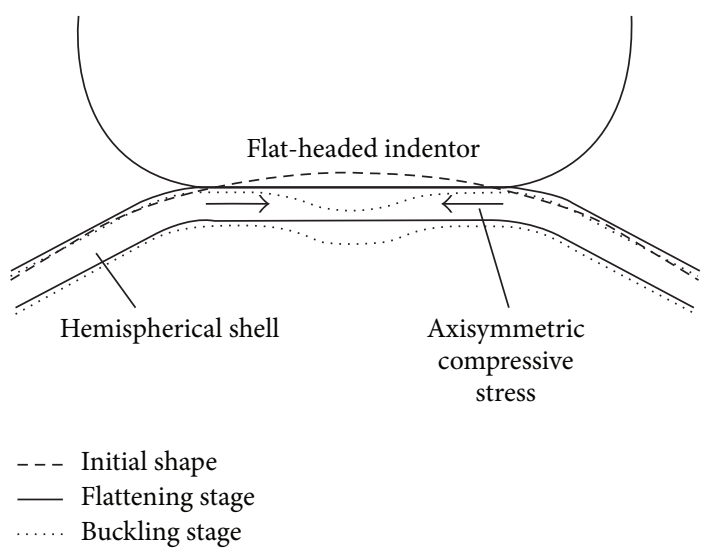

FIGURE 5: Deformation behavior in early indentation stage of SF.

indentor. The force drop just before the sharp increase is not clearly seen. Variation in the force exhibits a plateau for the range from 10 to $18 \mathrm{~mm}$ stroke after a slight peak. This kind of force plateau is seen also in axial collapse of honeycombs and tubes, where the cyclic buckling lobes are formed. The stroke of the force plateau is not long; however, this kind of feature is often preferred for the shock absorbing component.

In the case of SF, the apex part of the hemispherical shell becomes flat increasing the contact area to the indentor as drawn by the solid line in Figure 5. The in-plane compressive stress arises. Then the indentation force rapidly increases as shown in Figure 4, because the deformation is constrained by the flat bottom of the indentor. When the stress reaches a certain critical level, the material begins to buckle. Such force increase is usually preferable in view of the improvement in the energy absorption performance per structural mass.
When the stress reaches a certain critical level, the material begins to buckle and then the bending deformation also contributes the deformation and the force level. The shell top flattens at about indentation stroke of $1 \mathrm{~mm}$. The illustration of buckling is that in $2 \mathrm{~mm}$ stroke as observed in Figure 3(d).

The force decreases showing a plateau after peak, whose level is comparable with that of SS, because both deformation modes are similar. The rounded portion formed by buckling finally becomes flat by the stretching of the periphery during the stage of sharp force increase. Comparing the general force variation for two shell shapes, it is greater in flat top shell than that in hemispherical one, because the structural rigidity is higher in flat top shell.

The consumed energy in indentation is recognized as an index of energy absorption performance. Generally speaking, deformation for energy absorption requires some space. The 


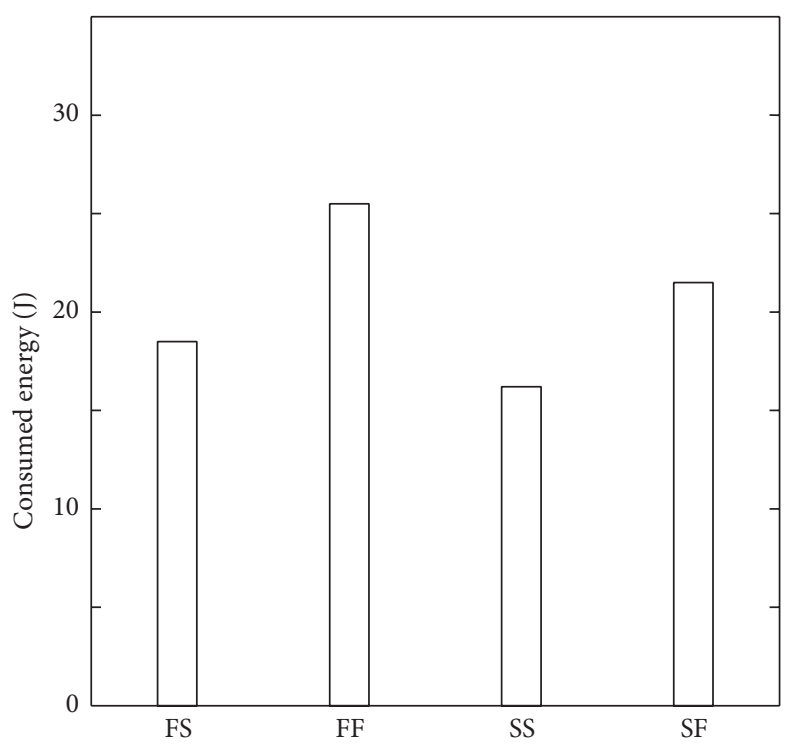

FIGURE 6: Summary of consumed energy in indentation stroke equal to shell height.

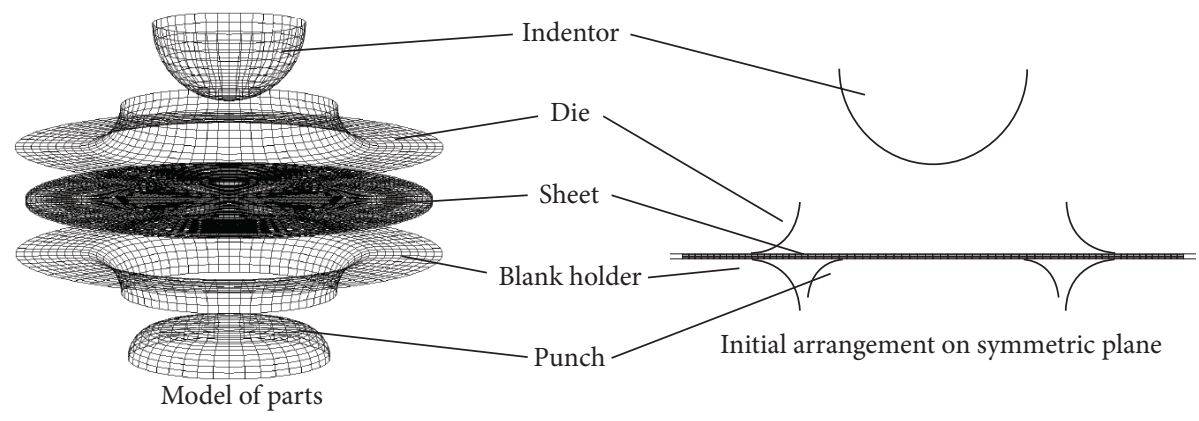

Figure 7: Numerical model (FS: flat top shell and hemispherical indentor).

admissible space is usually considered to be the inner space of the shell. Hence, it may be appropriate to calculate the consumed energy in the travel length of the indentor equal to the shell height of $10 \mathrm{~mm}$. The consumed energy $E_{C}$ is defined in the form

$$
E_{C}=\int_{0}^{S_{h}} F(x) d x
$$

where $F$ is the indentation force and $S_{h}$ is the shell height.

The consumed energy is presented in Figure 6 for each deformation condition. It is largest in the case of FF. The increase ratio of the energy is about $30 \%$ when the hemispherical indentor is replaced by a flat-headed one in both shell shapes. Further, the performance of SF is comparable with the case of FS. This implies that the energy absorption performance is affected by not only the product shape but also the crushing mode, which remarkably depends on the indentation manner.

\section{Numerical Simulation}

4.1. Computational Conditions. The numerical model is shown in Figure 7, which corresponds to the experimental condition FS (combination of flat top shell and hemispherical indentor). The left figure is the general view of the 3D model describing elements of the tools and the sheet. The right one indicates their initial arrangements on symmetric plane. Dimensions of the numerical model are similar to those in experiment. The tools are described with shell elements whose mechanical property is assumed to be rigid. The elements of the sheet are described with the 14,336 hexahedron solid elements and the number of nodes is 18,245 . The number of the layer through thickness is 4 . This division number was used in the numerical simulations of other sheet forming processes by one of the authors, where the deformation behavior was appropriately predicted $[17,18]$. In computation, the shell forming is first processed by the punch, while the indentor is stationary. After the shell forming, the punch moves backward $40 \mathrm{~mm}$. Applied nodal forces on the shell surface contact with the punch decrease and eventually become 0 during the unloading process. Thus the shell is supported by the blank holder and the die. Subsequently, the indentor travels downward toward the shell.

The dynamic explicit finite element solver DYNA3D (public domain version) was used [19]. In order to apply the code to the quasistatic deformation, the speed scaling 

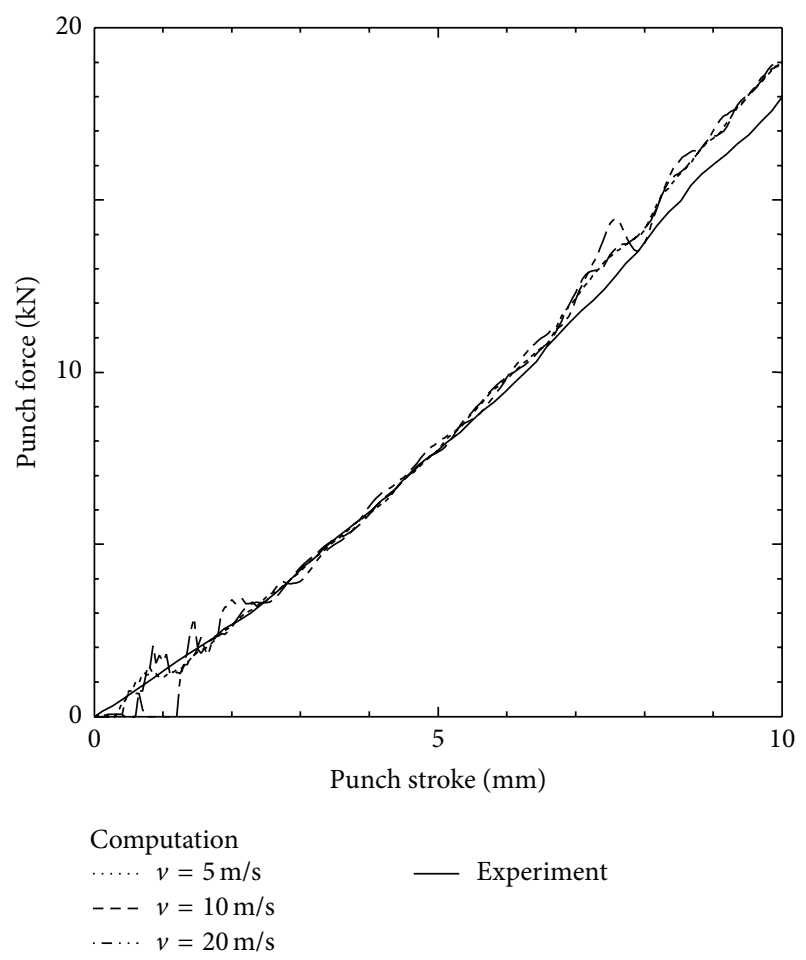

(a) Punch force-stroke curves

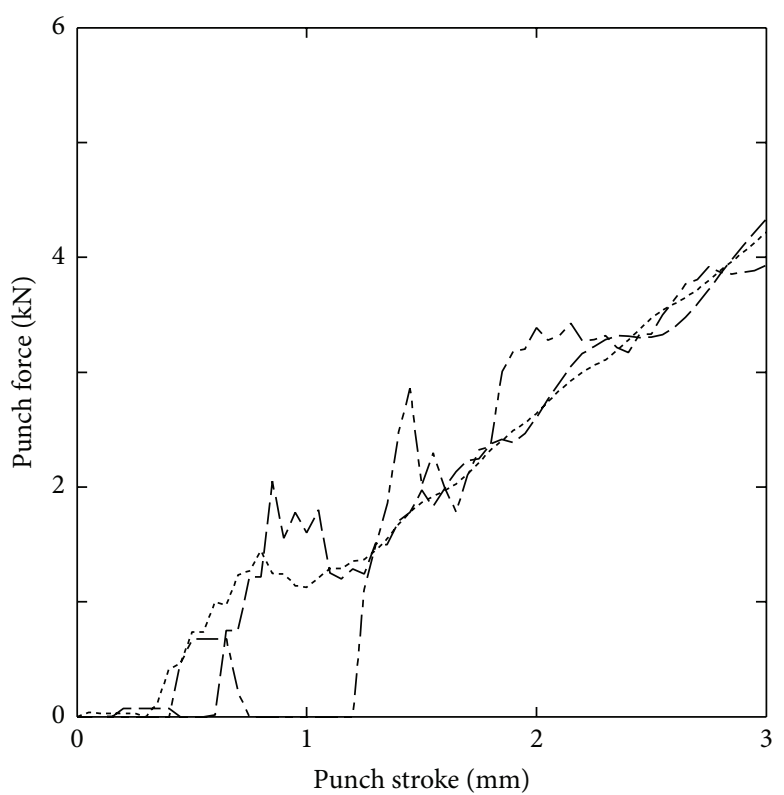

Computation
$\ldots \ldots v=5 \mathrm{~m} / \mathrm{s}$
$---v=10 \mathrm{~m} / \mathrm{s}$
$\ldots \quad v=20 \mathrm{~m} / \mathrm{s}$

(b) Enlarged view of early forming stage

FIGURE 8: Punch force-punch stroke curves in press forming of flat top shell and calculated ones under 3 speed conditions $(n=0.15, C=$ $383 \mathrm{MPa})$.

technique is commonly introduced for this kind of solver. The technique is very effective for saving the computational time, where the tool speed is set to be much faster than the actual one. It is available unless the effect of material inertia arises. Therefore, the tool speed was determined before the calculation of indentation in the present study.

The isotropic elastic plastic material with $n$th power strain hardening property was assumed for the sheet material. The friction of all interfaces was ignored. The punch speed was set to 5,10 , or $20 \mathrm{~m} / \mathrm{s}$ for trial in speed scaling. The calculated punch force-stroke curves in the flat top shell forming are shown in Figure 8 together with the experimental one. The nodal forces of the punch in the moving direction are summed to obtain the punch force. General trend seems similar for these three speeds, all of which generally agree with the experimental result. However, in the enlarged view of the early stage, the punch force indicates zero from 0.75 to $1.2 \mathrm{~mm}$ stroke in the case of $20 \mathrm{~m} / \mathrm{s}$ punch speed, which means that the separation of the sheet from the punch occurs due to the excessive inertia of the material. Further, overshooting force at around $2 \mathrm{~mm}$ stroke is observed, which may also be explained by the same reason. On the other hand, the difference between 5 and $10 \mathrm{~m} / \mathrm{s}$ conditions seems rather small. Therefore, the inertia effect under $10 \mathrm{~m} / \mathrm{s}$ punch speed can be practically ignored; thus the tool speed was determined $10 \mathrm{~m} / \mathrm{s}$ in view of the computational efficiency. The difference between the calculated shell height and the prescribed one due to spring back was as small as $0.05 \mathrm{~mm}$. The effect is considered practically negligible.
4.2. Computational Results and Discussions. The indentation force-stroke curves in computation and experiment are shown in Figure 9 for FS condition. The force variation is qualitatively predicted with the rapid increase nearing the end of the stroke due to the stretch of the shell wall. However, the difference between these two conditions is obvious, where the calculated force is greater than that in experiment. This may be due to the fact that the plastic hardening property of the material is assumed to be isotropic. Considering that the loading direction in the indentation process is opposite to that in the forming process and the rounded part bent by the die profile recovers to be flat, the Bauschinger effect of the material may arise. A series of tensile and compressive operations of soft A5052 aluminum alloy revealed that the Bauschinger effect in stress reversal appears as the plastic strain increases [20]. Consequently, the kinematic hardening plasticity may be more appropriate than the isotropic one. The kinematic linear hardening plasticity as shown in Figure 10 is available in the solver code used here. The parameter $\beta$ is set to 1 in isotropic hardening and becomes less than 1 in kinematic hardening. Considering that the test material used here is the $1 / 4$ hard or work-hardened one, the value 0 for the beta may be allowable.

The plastic property of the material has to be again determined considering the availability of linear hardening plasticity for the further calculation. For that purpose, it may be reasonable to compare the calculated press forming force with the experimental one conducting a parametric study changing the initial yield stress and the hardening modulus. 


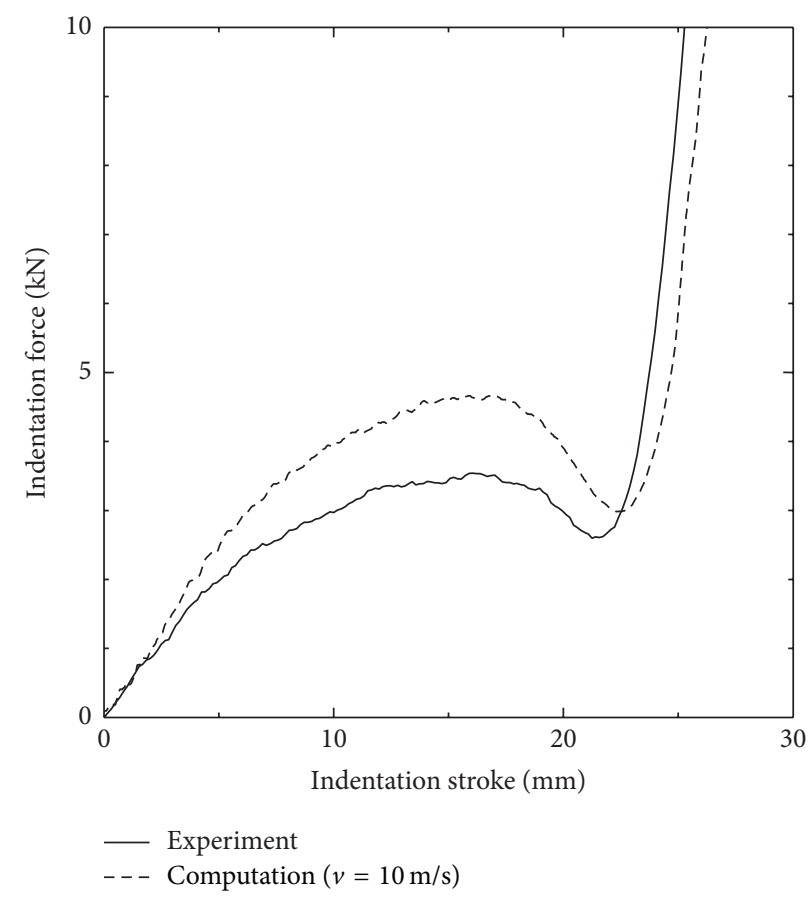

FIGURE 9: Indentation force-stroke curves in FS.

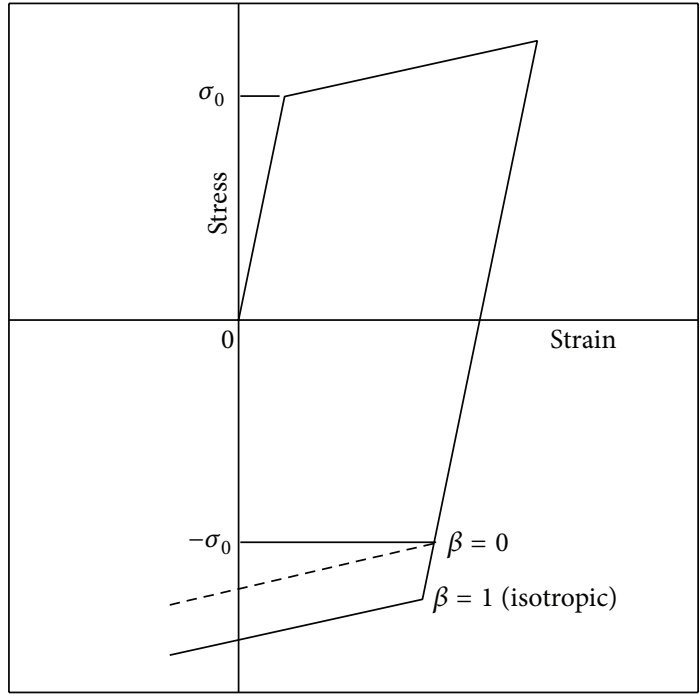

FIGURE 10: Kinematic linear hardening plasticity.

The forming force in the linear hardening property of $\sigma=$ $210+420 \varepsilon \mathrm{MPa}$ agrees well with the results in the experiment and in the computation with isotropic $n$th power hardening law as demonstrated in Figure 11. These determined plastic parameters are used in the further computations.

The force-stroke curves of indentation process for FS condition are drawn for $\beta=0,0.5$, or 1 in Figure 12. The curve with $\beta=0$ is generally close to the experimental result over the most indentation stroke, though the calculated force at concavity part is about $20 \%$ smaller than that in experiment. In the further computation, the parameter $\beta$ is set to 0 .

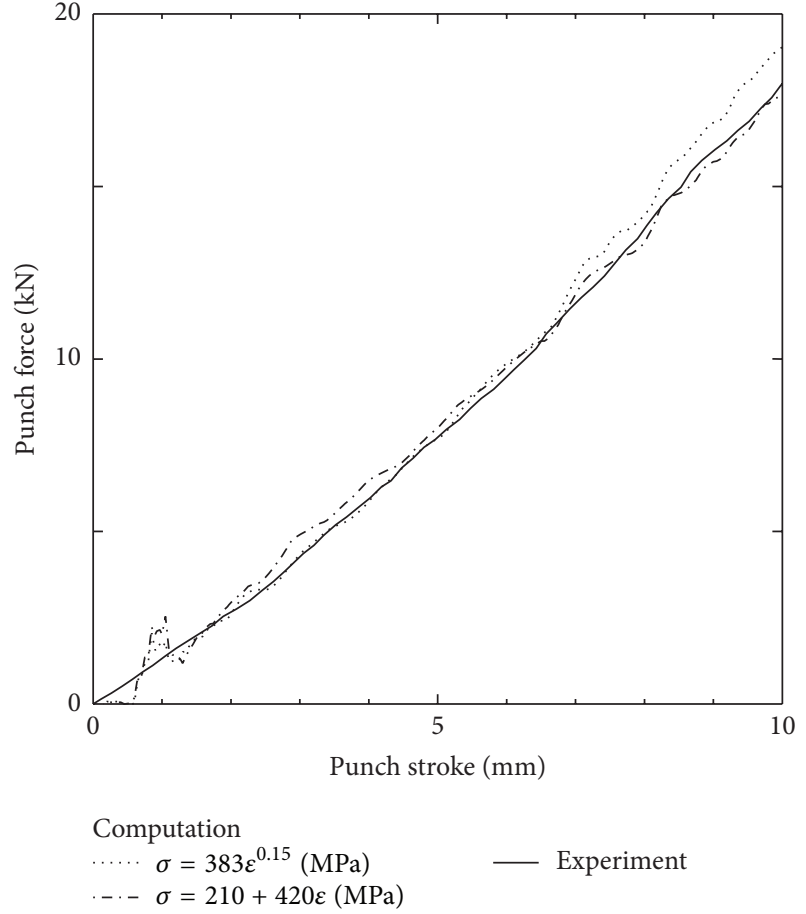

FIgURE 11: Punch force-stroke curves in computation for two mechanical properties.

Progressive deformation patterns are exhibited in Figure 13 for each condition. The press forming process is the deformation Stages 1 3, where the forming punch is also drawn. The indentation process is Stages $4 \sim 9$, where the indentor is drawn. The deformation patterns predicted by 


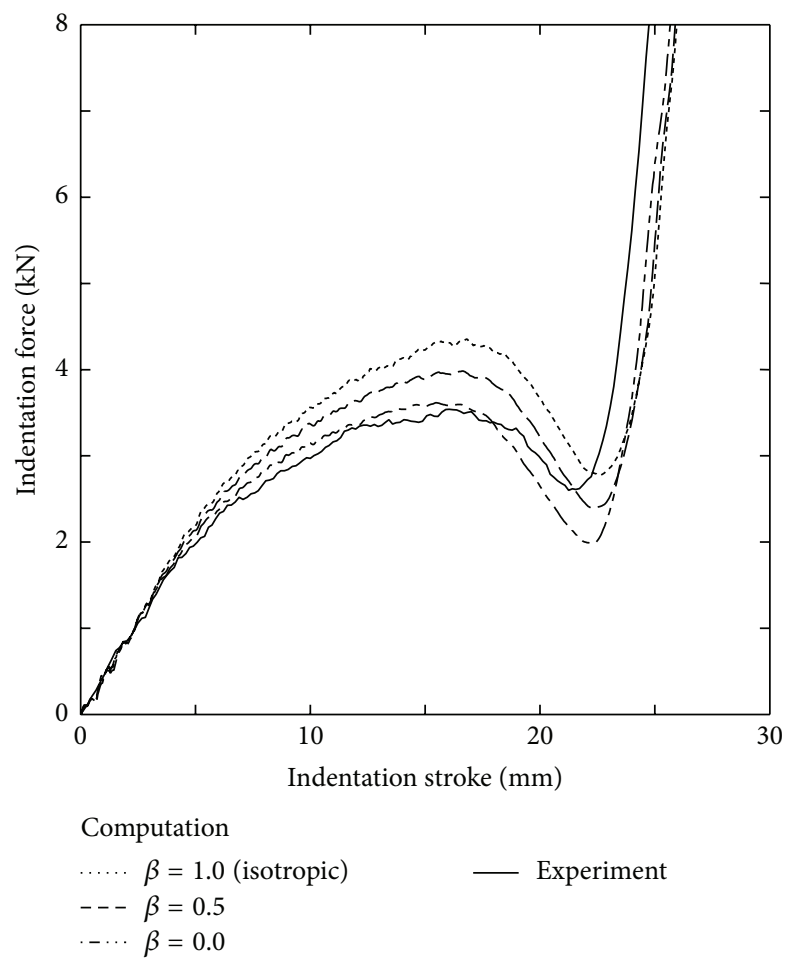

FIGURE 12: Indentation force-strokes curves for isotropic or kinematic hardening property.

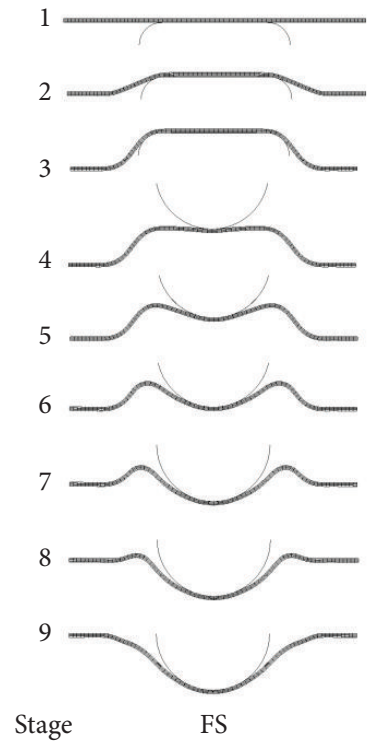

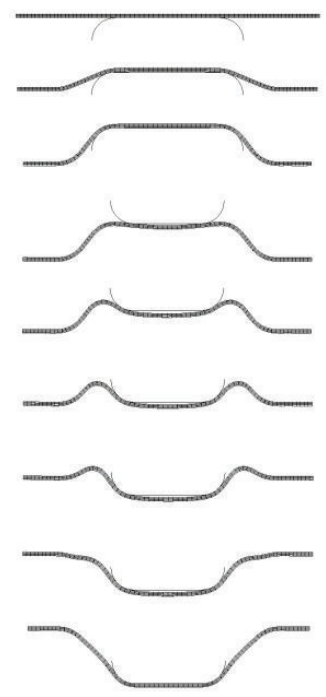

FF

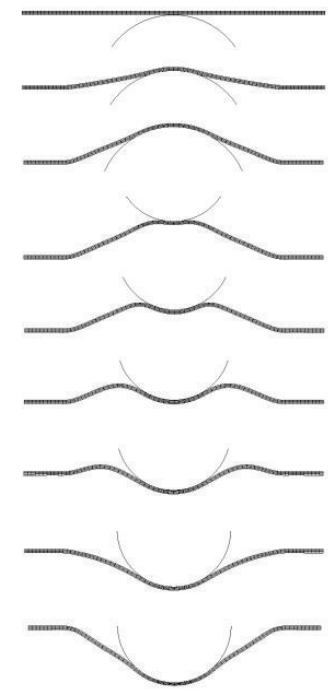

SS

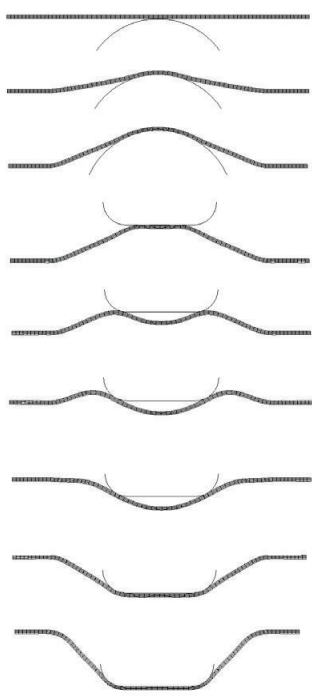

$\mathrm{SF}$

FIGURE 13: Progressive deformation patterns in computation, Stages 1 3: forming process, Stages 4 9: indentation process; indentation stroke: 1, 5, 10, 15, 20, $25 \mathrm{~mm}$ (Stages 4 9).

computation are well consistent with the experimental result for all conditions. Figure 14 shows the indentation forcestroke curves obtained by computation and experiment for each condition. Steeper force increase at the incipient stage is successfully simulated for FF and SF conditions (flat-headed indentor), comparing with that for FS and SS conditions (hemispherical indentor). The force-stroke curve for the case of FS in Figure 14(a) is the same as that in Figure 12 except for the scale of vertical axis, in which the force is adequately predicted in the most indentation stroke as mentioned. The calculated force of FF is slightly greater than that of the corresponding experiment in Figure 14(b).

Further, the less variation in indentation force before the rapid force increase at the final phase is also successfully 


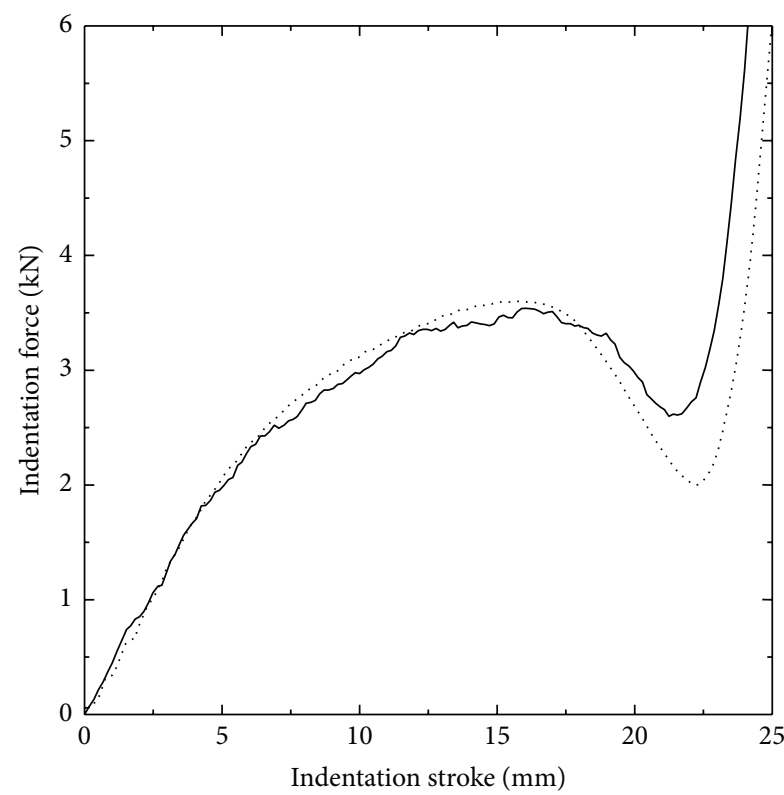

- Experiment

..... Computation

(a) FS

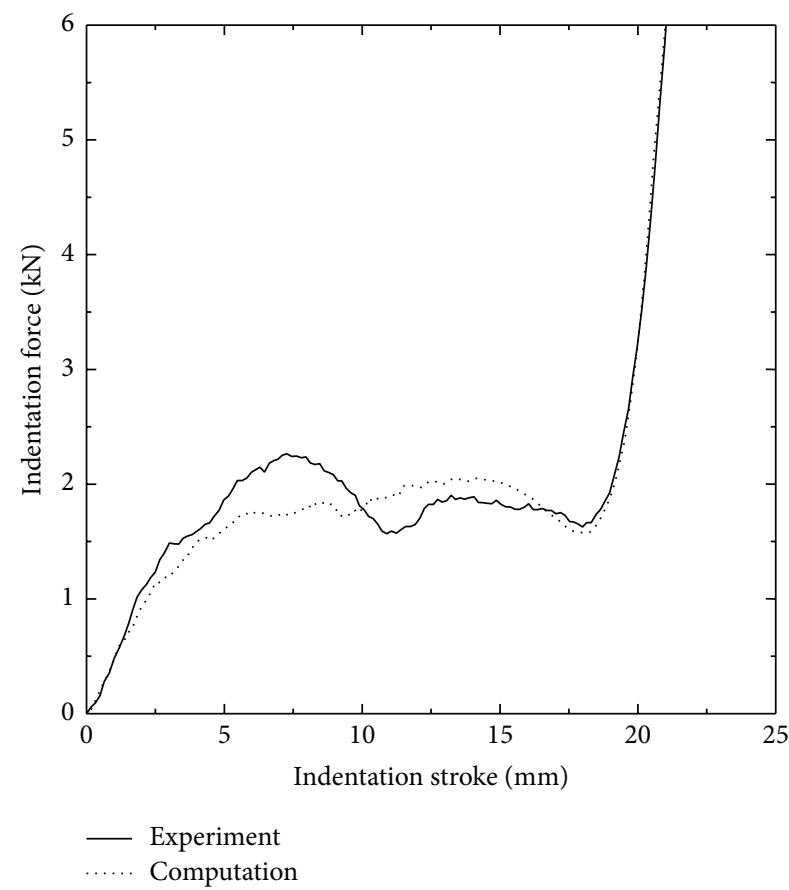

(c) SS

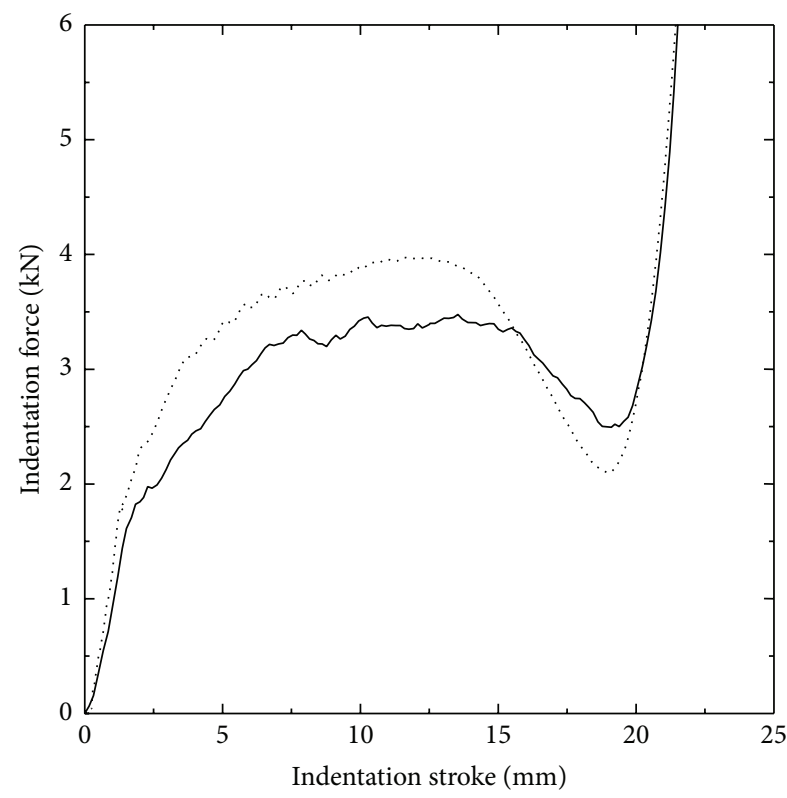

- Experiment

..... Computation

(b) FF

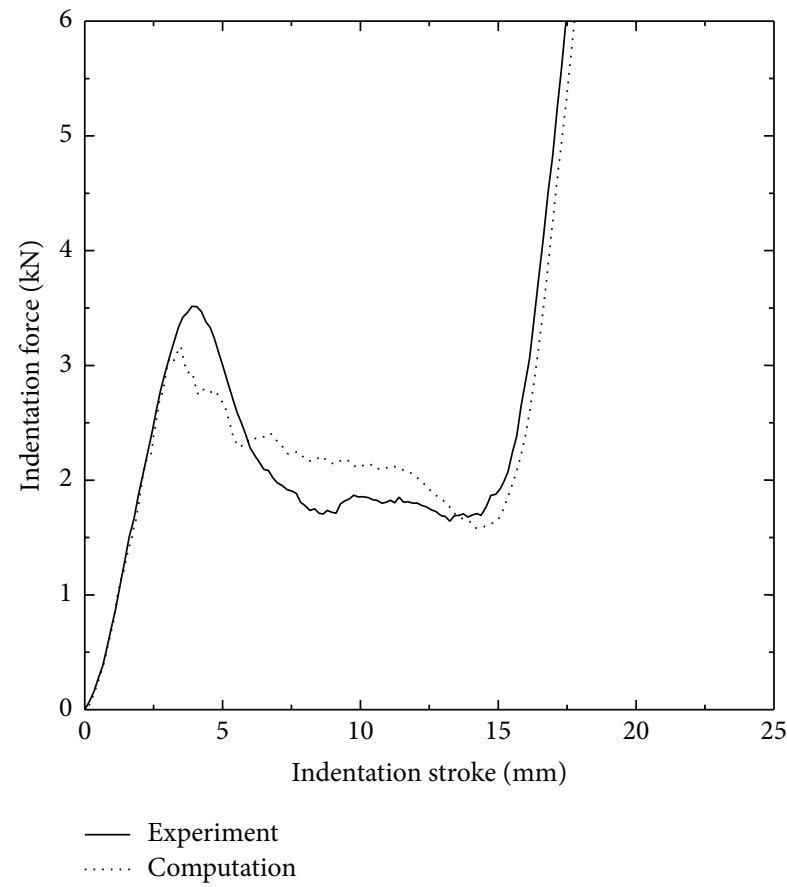

(d) $\mathrm{SF}$

FIGURE 14: Indentation force-stroke curves obtained in experiment and computation for various indentation conditions.

simulated in the case of SS as shown in Figure 14(c). It is noted that the initial peak force followed by the gradual decrease in the case of SF is also properly predicted as shown in Figure 14(d). This phenomenon is because of the buckling of the material in front of the indentor head as demonstrated in Stages 4 and 5 of SF in Figure 13.

The consumed energy by the deformation is shown in Figure 15, whose value corresponds to the area under the 


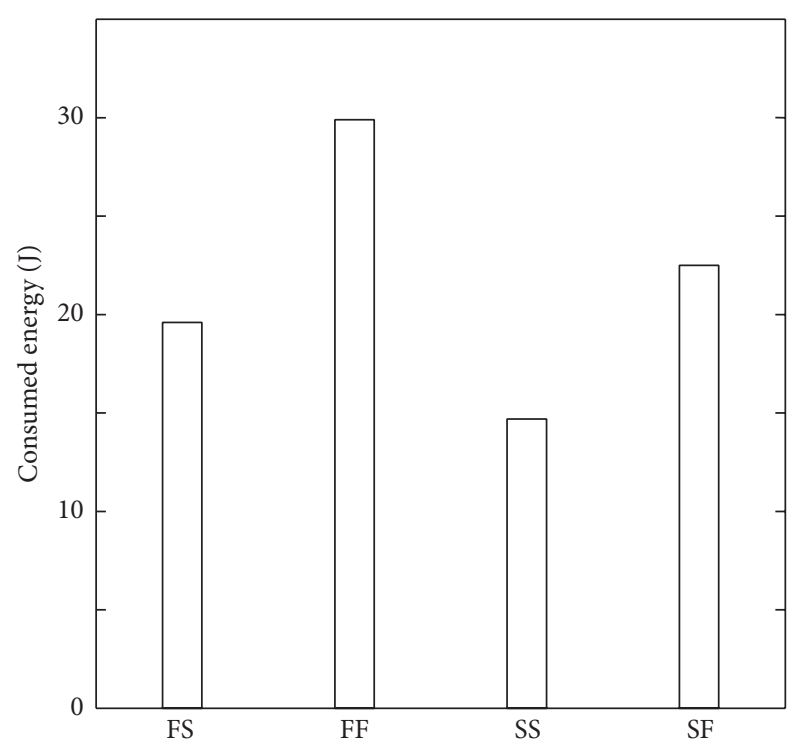

FIGURE 15: Calculated consumed energy in indentation stroke equal to shell height.

indentation force-stroke curve. The predicted values are almost comparable with those in experiment. However, considering that the friction is ignored in calculation and the linear hardening model is used, there is a room for improvement.

\section{Conclusions}

Flat top and hemispherical aluminum alloy A5052 shells fabricated by the press forming were deformed by the flator hemispherical-headed indentor. Deformation patterns and characteristic of the indentation force were investigated. Energy absorption performance in indentation deformation of the shells was estimated for indentation stroke corresponding to the shell height. Numerical simulation was also carried out.

The energy absorbing performance of the shell was greater in the case of flat-headed indentor than in the case of hemispherical one. Axisymmetric plastic buckling deformation under in-plane axisymmetric compressive stress field was found very effective for the increase of the resistance to collapse. When the hemispherical shell was deformed by similar shaped indentor, less force variation was achieved due to the appropriately generated mobile plastic hinge. The deformation patterns obtained in experiment were well simulated by the finite element calculation. The accuracy in prediction of indentation force was improved by introducing the kinematic hardening plasticity in the material constitutive model, on the other hand, the force was overestimated when isotropic plasticity was applied. Bauschinger effect is one of the considerations in designing the press formed parts used as energy absorbing component.

In the present study, several basic characteristics in the indentation behavior were revealed for the simple collapse conditions; they may provide some helpful guidelines in designing the collapsible parts and structures.

\section{Conflict of Interests}

The authors declare that there is no conflict of interests regarding the publication of this paper.

\section{References}

[1] A. A. A. Alghamdi, "Collapsible impact energy absorbers: an overview," Thin-Walled Structures, vol. 39, no. 2, pp. 189-213, 2001.

[2] A. G. Olabi, E. Morris, and M. S. J. Hashmi, "Metallic tube type energy absorbers: a synopsis," Thin-Walled Structures, vol. 45, no. 7-8, pp. 706-726, 2007.

[3] C. Bisagni, "Crashworthiness of helicopter subfloor structures," International Journal of Impact Engineering, vol. 27, no. 10, pp. 1067-1082, 2002.

[4] A. K. Pickett, T. Pyttel, F. Payen et al., "Failure prediction for advanced crashworthiness of transportation vehicles," International Journal of Impact Engineering, vol. 30, no. 7, pp. 853-872, 2004.

[5] M. Zupan, C. Chen, and N. A. Fleck, "The plastic collapse and energy absorption capacity of egg-box panels," International Journal of Mechanical Sciences, vol. 45, no. 5, pp. 851-871, 2003.

[6] N. K. Gupta, G. L. Prasad, and S. K. Gupta, "Axial compression of metallic spherical shells between rigid plates," Thin-Walled Structures, vol. 34, no. 1, pp. 21-41, 1999.

[7] N. K. Gupta and Venkatesh, "Experimental and numerical studies of dynamic axial compression of thin walled spherical shells," International Journal of Impact Engineering, vol. 30, no. 8-9, pp. 1225-1240, 2004.

[8] P. K. Gupta and N. K. Gupta, "A study of axial compression of metallic hemispherical domes," Journal of Materials Processing Technology, vol. 209, no. 4, pp. 2175-2179, 2009.

[9] M. Shariati and H. R. Allahbakhsh, "Numerical and experimental investigations on the buckling of steel semi-spherical shells under various loadings," Thin-Walled Structures, vol. 48, no. 8, pp. 620-628, 2010.

[10] X. L. Dong, Z. Y. Gao, and T. X. Yu, "Dynamic crushing of thinwalled spheres: an experimental study, International Journal of Impact Engineering, vol. 35, no. 8, pp. 717-726, 2008.

[11] A. A. A. Alghamdi, "Reinversion of aluminium frustra," ThinWalled Structures, vol. 40, no. 12, pp. 1037-1049, 2002.

[12] M. Yamashita and M. Gotoh, "Impact behavior of honeycomb structures with various cell specifications-numerical simulation and experiment," International Journal of Impact Engineering, vol. 32, no. 1-4, pp. 618-630, 2005.

[13] W. Abramowicz and N. Jones, "Dynamic progressive buckling of circular and square tubes," International Journal of Impact Engineering, vol. 4, no. 4, pp. 243-270, 1986.

[14] M. Yamashita, H. Kenmotsu, and T. Hattori, "Dynamic axial compression of aluminum hollow tubes with hat cross-section and buckling initiator using inertia force during impact," ThinWalled Structures, vol. 50, no. 1, pp. 37-44, 2012.

[15] M. Yamashita, H. Kenmotsu, and T. Hattori, "Dynamic crush behavior of adhesive-bonded aluminum tubular structureexperiment and numerical simulation," Thin-Walled Structures, vol. 69, pp. 45-53, 2013.

[16] E. Siebel, "Niederhalterdruck beim tiefziehen," Stahl und Eisen, vol. 74, no. 3, pp. 155-158, 1954.

[17] M. Yamashita, T. Hattori, and N. Nishimura, "Numerical simulation of sheet metal drawing by Maslennikov's technique," 
Journal of Materials Processing Technology, vol. 187-188, pp. 192196, 2007.

[18] M. Yamashita, T. Hattori, K. Yamada, and N. Nishimura, "Frictional effect on deformation behavior in incremental sheet forming," Steel Research International, vol. 81, no. 9, pp. 926-929, 2010.

[19] J. O. Halliquist, DYNA3D Theoretical Manual, 1989.

[20] S. Tamura, S. Sumikawa, T. Uemori, H. Hamasaki, and F. Yoshida, "Experimental observation of elasto-piasticity behavior of type 5000 and 6000 aluminum alloy sheets," Materials Transactions, vol. 52, no. 5, pp. 868-875, 2011. 

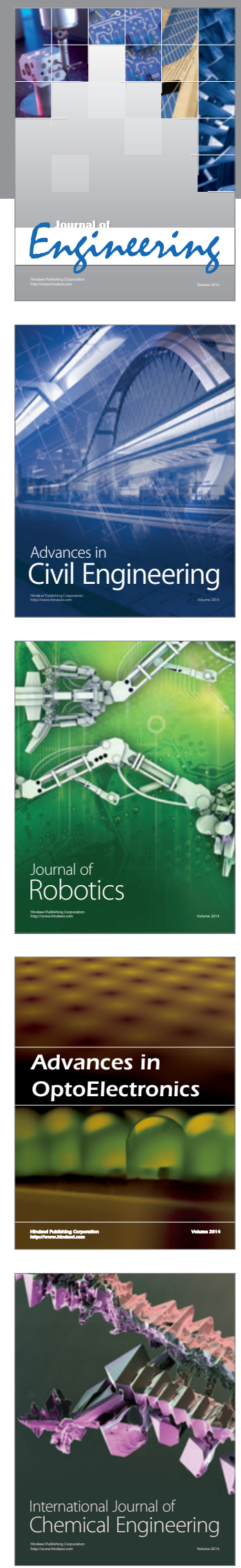

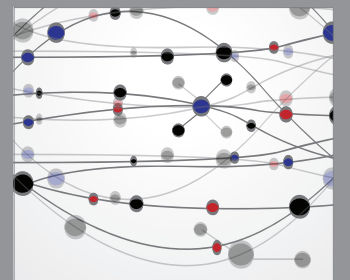

The Scientific World Journal
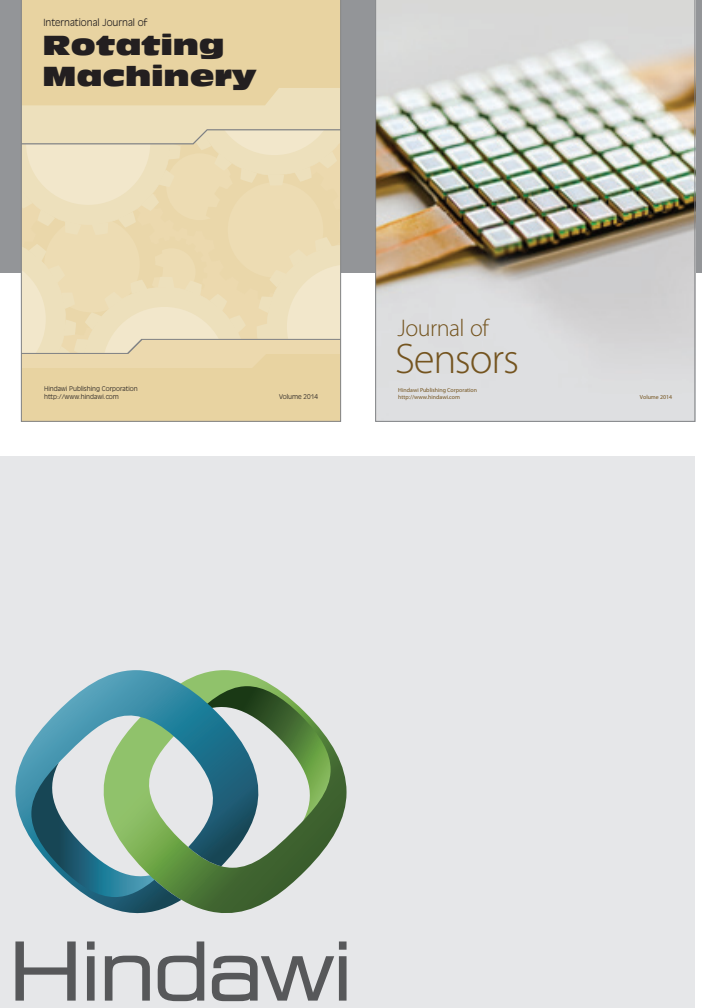

Submit your manuscripts at http://www.hindawi.com
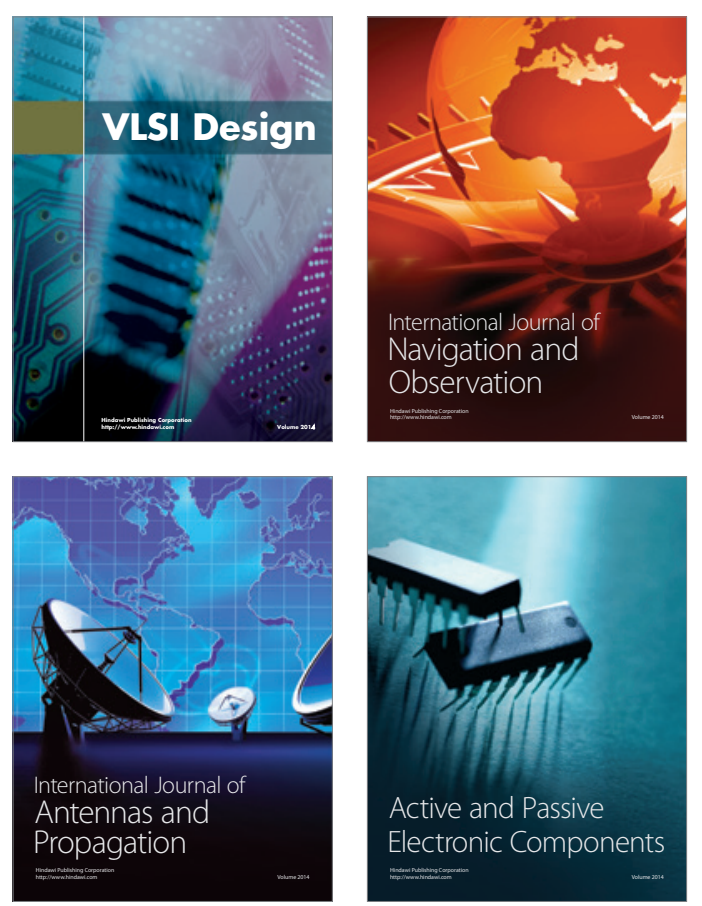
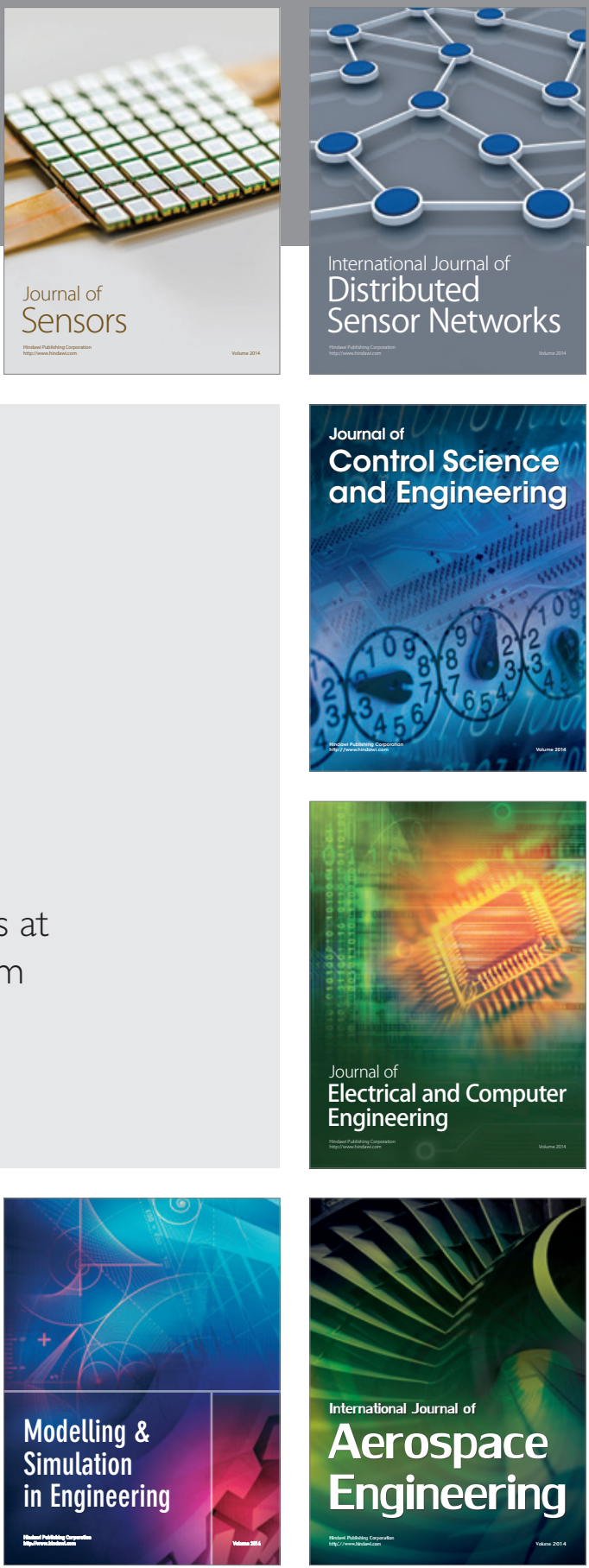

Journal of

Control Science

and Engineering
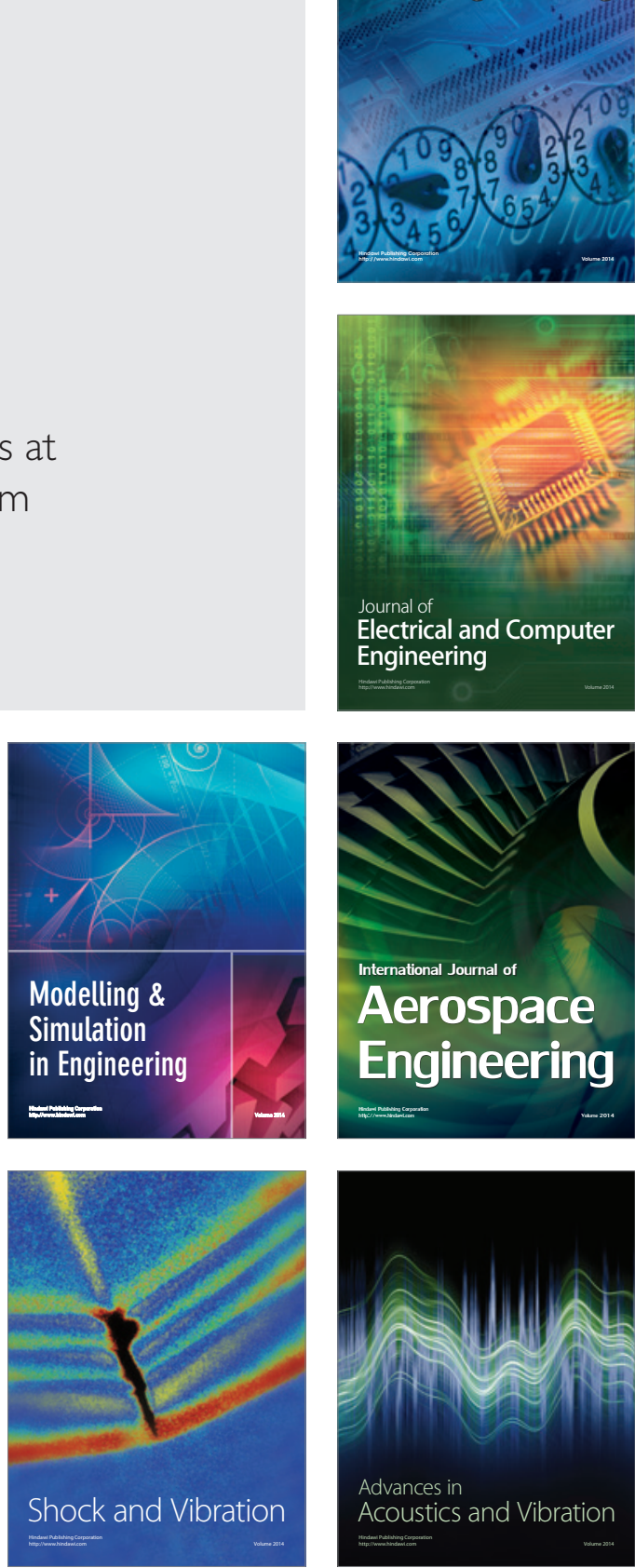